\title{
Visualization of Light Elements at Ultrahigh Resolution by STEM Annular Bright Field Microscopy
}

\author{
Eiji Okunishi, Isamu Ishikawa, Hidetaka Sawada, Fumio Hosokawa, Madoka Hori \\ and Yukihito Kondo
}

Electron Optics Division, JEOL Ltd., 1-2 Musashino Akishima 3-chomeTokyo, 196-8558, Japan.

In the field of materials sciences such as studies on ceramics, semi-conducting material and metals, role of light elements is important, because it is one of mainly composing elements or determiner of character i. e. dopants. The light elements at high resolution have been observed by ultrahigh voltage electron microscopy or aberration corrected electron microscopy in Transmission Electron Microscopy (TEM), since the visualization of light requires highly resolving power. Recently, a high angle annular dark field (HAADF) scanning transmission electron microscopy (STEM) has become widely used in this field because of high-resolution capability and easily interpretable image contrast, which is roughly proportional to square of atomic number $Z\left(Z^{2}\right)$. However, the HAADF image sometimes gives lack of light element because of excess contrast originated from $Z^{2}$, when the specimen contains the light and heavy elements. The TEM bright field imaging gives an image contrast roughly proportional to the $\mathrm{Z}$, when the specimen is thin enough to be able to apply the "thin film approximation'. We have examined to apply an STEM annular bright field (ABF) imaging, which is equivalent to TEM hollow cone illumination imaging technique [1-2], to the oxide or nitride samples for simultaneous visualization of light and heavier elements. According to the article on hollow cone illumination in TEM [2], the contrast transfer in ABF expected to give better resolution than conventional BF STEM and to give non-oscillating contrast transfer, which gives easily-interpretable images unlike the BF STEM. This paper reports characteristics and the experimental result of the $\mathrm{ABF}$ imaging technique.

Experiments were performed with a new $200 \mathrm{kV}$ microscope (JEM-ARM200F) equipped with an annular bright field and dark field detectors as well as a spherical aberration correction system for STEM [3]. Figure 1 shows a scheme for our experiment. This experimental configuration enables us to perform a simultaneous acquisition of annular dark and bright field images. The convergent angle of incident beam is limited with the aperture in condenser lens system. The inner and outer acceptance angle for HAADF image is limited with the camera length and size of the HAADF detector. Those for ABF image is limited with the camera length, size of the ABF detector and the size of preventing disc placed above the bright field detector.

Figures 2 (a), (b) and (c) show the HAADF, conventional BF and ABF images of $\mathrm{SrTiO}_{3}(001)$. And the model of $\mathrm{SrTiO}_{3}(001)$ is illustrated in Fig. 2 (d). The experimental parameters for the observation are listed as follows. Detecting angle for HAADF, BF and ABF were $68-200 \mathrm{mrad}, 0-22 \mathrm{mrad}, 11-22$ mrad, respectively. The probe current and size were $24 \mathrm{pA}$ and $0.1 \mathrm{~nm}$. If we focus on the site of oxygen, the oxygen is invisible in the HAADF image (Fig. 2(a)) and slightly visible in the BF image (Fig. 2(b)). While in the ABF image (Fig. 2(c)), the oxygen is clearly visible. Additional experiments of focal dependency were performed with the through-focal series of HAADF, BF, and ABF image. The results confirmed that $\mathrm{ABF}$ images gave non-oscillating contrast transfers at various foci like HAADF images different from $B F$ images.

We have examined other samples. The results $\mathrm{HAADF}$ and $\mathrm{ABF}$ for $\mathrm{Fe}_{3} \mathrm{O}_{4}$ and $\mathrm{Si}_{3} \mathrm{~N}_{4}$ are shown in Figures 3 (a), 3 (b), 3 (a') and 3 (b'). Nitrogen and oxygen are clearly visible in corresponding sites in $\mathrm{ABF}$ images but invisible in HAADF. The ABF imaging enables us to see light atomic sites directly. Furthermore, simultaneous acquisition of HAADF and ABF gives us the good correspondence of atomic site, since the HAADF imaging is incoherent imaging, which gives reliable position of atomic site. 


\section{References}

[1] J. Fertig and H. Rose Optik, 54, 165 (1979).

[2] W. O. Saxton et al, Optik, 49, 505 (1978).

[3] I. Ishikawa et al, Proceedings of this conference.

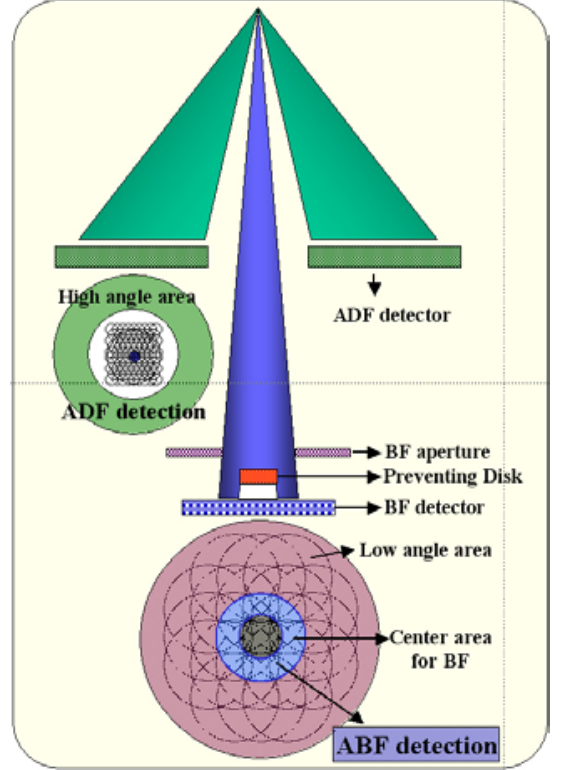

Fig. 1 Experimental set up for HAADF and $\mathrm{ABF}$ imaging.
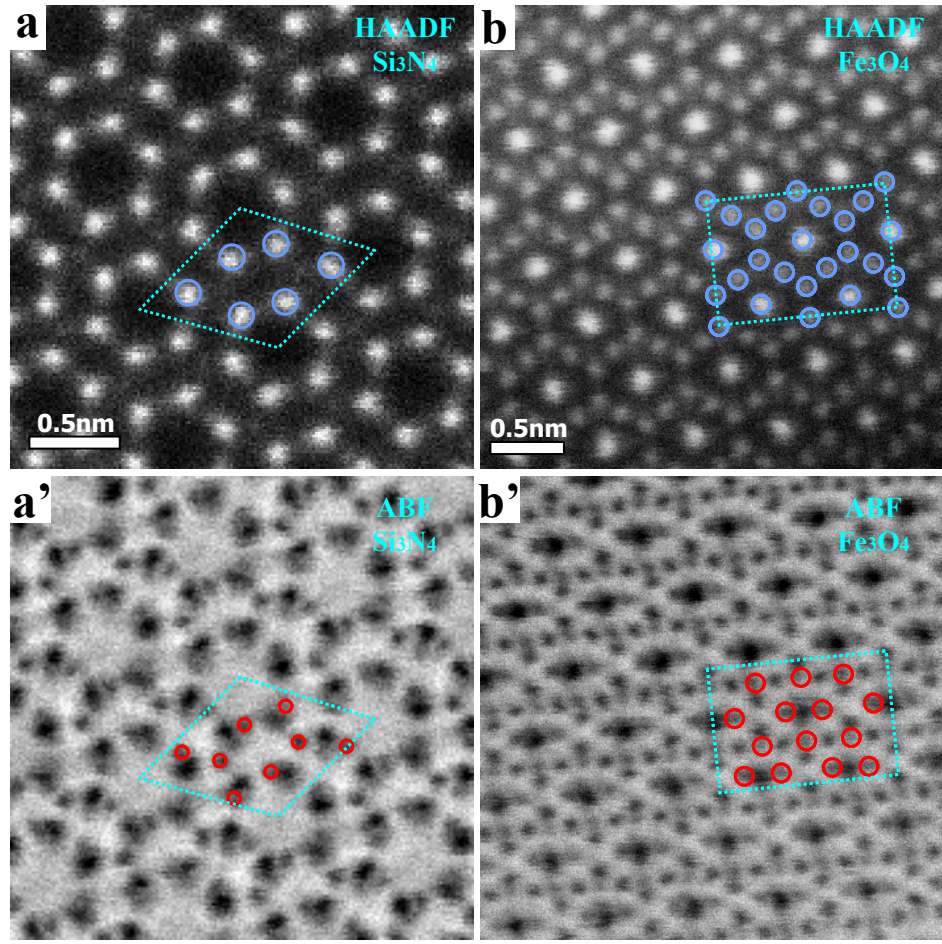

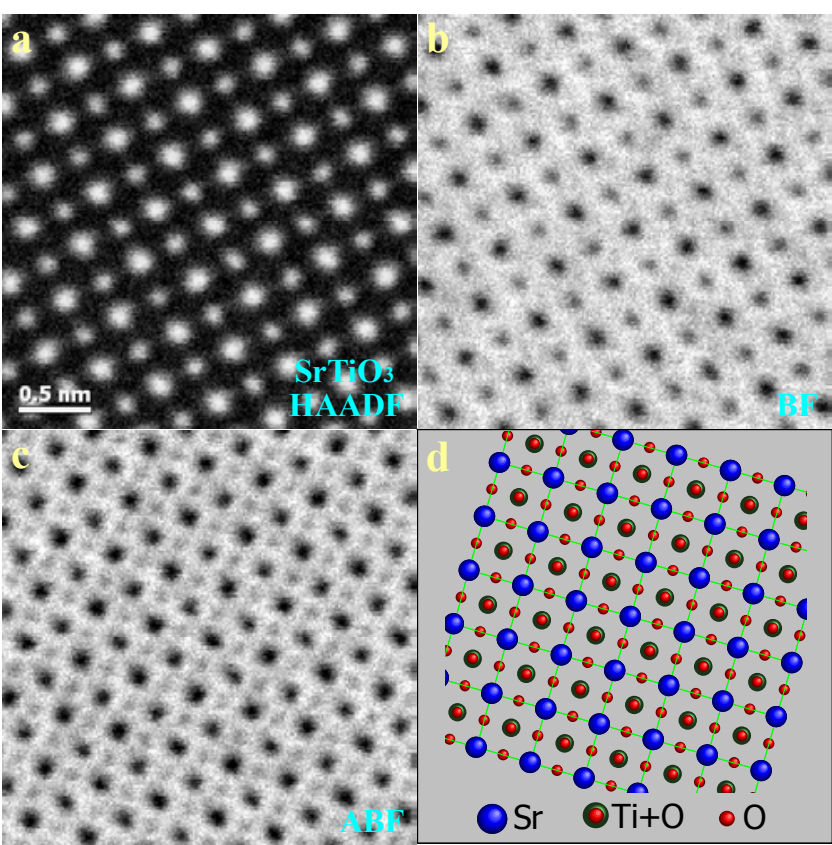

Fig. 2

(a) HAADF and (b) conventional BF image, (c) ABF image and (d) Structure model of $\mathrm{SrTiO}_{3}[001]$ direction. Oxygen position is clearly visible in $\mathrm{ABF}$ image.

Fig. 3

(a) HAADF and (a')ABF images of $\mathrm{Si}_{3} \mathrm{~N}_{4}$, (b) HAADF and (b') $\mathrm{ABF}$ images of $\mathrm{Fe}_{3} \mathrm{O}_{4}$. light atom positions are indicated by red circles, heavy atoms are in blue circles in the inset of images. 